\title{
Alfabetización informacional a través de Moodle
}

\author{
Gabriela Fernanda Cabrera Rossi \\ Instituto Universitario Asociación Cristiana de Jóvenes - IUACJ, Uruguay.
}

COMUNICACIÓN

\section{Resumen}

Se describe la experiencia de la Biblioteca "Dr. Claudio Williman" de la Facultad de Educación Física del Instituto Universitario de la Asociación Cristiana de Jóvenes (IUACJ), en el desarrollo de cursos que contribuyen a la mejora de la calidad formal de los trabajos académicos. Se presentan distintos tipos de cursos impartidos por Biblioteca de carácter semipresencial, los cuales generan créditos académicos y son desarrollados bajo la plataforma Moodle. Se describe el proceso de integración del IUACJ a Moodle como herramienta educativa facilitadora para el desarrollo y organización de los cursos. Se analiza la contribución del bibliotecólogo, como gestor de información, evaluando recursos para luego ponerlos a disposición de la comunidad académica de estudiantes, proporcionando acciones que contribuyan a consolidar a la Biblioteca en su rol de alfabetizador, favoreciendo la adquisición de conocimientos y habilidades de los usuarios. También se enumeraran los cursos dictados por la Biblioteca describiendo estructura, metodología aplicada y recursos didácticos.

Palabras clave

Enseñanza virtual; Bibliotecas universitarias ; Plataformas de aprendizaje ; Moodle ; Alfabetización Informacional ; Instituto Universitario de la Asociación Cristiana de Jóvenes ; Uruguay

\section{Information literacy through Moodle}

\section{Abstract}

This articles described the experience of the Library "Dr. Claudio Williman " Faculty of Physical Education Institute (IUACJ) in the development of courses, that contribute to improving the formal quality of academic work. Different types of courses provided by the Library, which generate credits and are developed under the Moodle platform. The integration process described Moodle as an educational tool for facilitating the development and organization of the courses are presented. The contribution of the librarian is analyzed, as information manager, evaluating resources and then make them available to the academic community of students, providing actions that contribute to consolidate the Library in its role of literacy, favoring the acquisition of knowledge and skills users. Courses taught by the Library describing structure, applied methodology and teaching resources offered also enumerate.

Keywords

Virtual teaching ; University library ; Learning platform ; Moodle ; Information literacy ; Instituto Universitario de la Asociación Cristiana de Jóvenes ; Uruguay

\section{Introducción}

Actualmente, las universidades migran cada vez más del sistema tradicional de enseñanza basado en lo que podemos definir como un modelo pasivo en donde existe una simple transferencia de información del profesor al alumno, a un modelo participativo activo a través de la incorporación de los Entornos Virtuales de Aprendizaje, favoreciendo así la comunicación entre docente - alumno. 
Por esta razón y con el objetivo de generar espacios donde el alumno pueda contribuir a la construcción del conocimiento y donde el docente deje de ser el único transmisor de conocimientos, convirtiéndose en un guía o tutor del alumno en el proceso de adquisición de conocimiento, es que comienzan a obtener protagonismo la incorporación de los entornos virtuales como Moodle basados justamente en principios pedagógicos del contructivismo donde las personas construyen su propia conocimiento en forma activa, interaccionando con el medio y el construccionismo a través del cual el aprendizaje es efectivo cuando se logra construir algo que llega a otros. (Piaget, 1952).

\section{Moodle en el IUACJ}

Desde su creación en 1999 el IUACJ se propuso brindar actividades de enseñanza y alcanzar una sólida formación profesionales de Educación Física, Recreación y Deporte, mediante el desarrollo de programas educativos de grado, tecnicaturas y educación permanente. Actualmente el IUACJ dicta la Licenciatura en Educación Física, Recreación y Deporte, la Tecnicatura en Fitness, el curso de Técnico Deportivo y la Tecnicatura en Recreación y Tiempo Libre. La propuesta formativa es amplia, multidisciplinaria y con un fuerte componente práctico; por tal motivo a comienzos de 2010 el IUACJ solicita una consultoría con el objetivo de analizar, diseñar e implementar una propuesta con el fin de introducir paulatinamente las TICs como metodología que complemente la formación académica, organizando un conjunto de asignaturas bajo un formato semipresencial.

A partir de la consultoría el IUACJ definió políticas que favorecieron la integración de tecnologías que potenciaron el crecimiento de la institución y permitieron cumplir con los objetivos trazados.

De este modo es que se optó por la incorporación de software libre y de libre distribución, escogiendo Moodle para el desarrollo y dictado de cursos virtuales y semi presenciales. Los principales motivos que llevaron a su selección fue su facilidad de uso, su adaptabilidad a diferentes niveles de usuarios y su óptima relación costo rendimiento. A partir de la incorporación de la herramienta el personal docente y funcionarios del IUACJ recibieron capacitación en el uso de ambientes virtuales de aprendizaje. Siendo los objetivos específicos en cuanto a la formación:

- Analizar las características básicas de enseñanza y el aprendizaje en ambientes virtuales.

- Promover el uso de la plataforma virtual para apoyar las acciones de formación del IUACJ.

- Capacitar en el uso de la herramienta Moodle.

Finalizada la capacitación al grupo de docentes y funcionarios, se comenzaron a organizar cursossemipresenciales utilizando la plataforma. A partir de estas acciones es que la Biblioteca "Dr. Claudio Williman" del IUACJ, se incorpora al uso de Moodle y comienza a desarrollar e implementar distintos niveles de cursos semipresenciales académicos (algunos de ellos con créditos académicos) destinados a estudiantes y docentes de la institución.

\section{Integración de la plataforma Moodle a los cursos}

Si bien hasta el 2010 la Biblioteca brindaba cursos de formación de usuarios dirigidos sólo a estudiantes, los cursos básicamente cumplían con el objetivo de inducir al usuario en el uso y manejo de los recursos informativos de la Biblioteca, como por ejemplo el uso del catálogo, a través de sesiones denominadas "Conoce tu Biblioteca", no siendo las mismas de carácter obligatorio y presencial hasta el momento.

A partir de la incorporación de Moodle la Biblioteca amplía su oferta formativa de cursos ofreciendo la formación de usuarios en modalidad presencial y semipresencial. 
A partir de 2011, la Biblioteca además de dictar sesiones de formación de usuarios denominadas "Conoce tu Biblioteca", en modalidad presencial y semipresencial, comienza a impartir Ateneos Académicos y Seminarios a estudiantes de la Licenciatura en Educación Física, Recreación y Deporte (LEFRYD) los cuales a diferencia de las sesiones de formación de usuarios, generan créditos académicos. Los Ateneos Académicos correspondientes al Plan 2000, con actualización 2005 se enmarcaron dentro de estrategias de apoyo a la enseñanza propuestas por la Biblioteca y aprobado por la Dirección del IAUCJ y el Área de Investigación de la LEFRyD. Los contenidos vertidos en los cursos fueron la estructura de un trabajo académico, búsqueda, selección y evaluación de información académica, manejo de bases de datos (TIMBÓ), presentación de la norma ABNT (Asociación Brasilera de Normas Técnicas) norma bajo la cual se rige el IUACJ para citar y referenciar materiales impresos y electrónicos, y manejo de gestores bibliográficos. A diferencia de las sesiones de formación de usuarios (Conoce tu Biblioteca), los Ateneos Académicos, siempre fueron de carácter obligatorio y generaron créditos académicos ( 2 créditos), teniendo una carga horaria de 8 horas, de las cuales se dictaron 4 horas en forma virtual utilizando la plataforma Moodle.

La organización, desarrollo y dictado de clases de los Ateneos Académicos, estuvo a cargo de Bibliotecólogos de la Biblioteca del IUACJ, quienes previamente fueron capacitados en el uso de la plataforma. De esta manera, la Biblioteca se valió de las posibilidades que ofrece la plataforma

Moodle para el dictado de cursos semi presenciales, permitiendo a los alumnos trabajar en un ambiente colaborativo, que contribuyera al desarrollo de destrezas, habilidades informativas, y espíritu crítico, concretando el objetivo trazado por el Área de Investigación en coordinación con Biblioteca que centran su atención en la mejora de la calidad de los trabajos académicos elaborados por alumnos del IUACJ a través de un aprendizaje permanente que les permita desenvolverse en actual sociedad del conocimiento.

Las acciones descriptas contribuyeron a consolidar a la Biblioteca en su rol de alfabetizador informacional, favoreciendo el desarrollo de conocimientos y habilidades de los usuarios, administrando y gestionando la información contenida en diferentes formatos, medios y recursos (físicos, electrónicos y digitales) así como identificando, evaluando, recuperando, usando, generando y comunicando información de forma crítica, ética y legal.

A partir del 2011 el IUACJ modifica su Plan de Estudios, y a partir de ésta modificación considerando la necesidad de profundizar en algunos de los temas abordados durante los Ateneos Académicos, la institución decide incorporar al nuevo Plan de Estudios una nueva asignatura denominada Elaboración de Trabajos Académicos, de carácter transversal, dirigida a alumnos de primer año de la Licenciatura en Educación Física, Recreación y Deporte, durante el primer semestre, generando 2,5 créditos y contando con una carga horaria de 25 horas, dictada y coordianada por personal de Biblioteca. Para el desarrollo de esta asignatura se consideró beneficioso utilizar la plataforma Moodle, favoreciendo la interacción semipresencial con el alumno a través de la plataforma.

\section{Recursos didácticos aplicados}

A continuación se describe la organización de los contenidos didácticos de los cursos que dicta la Biblioteca utilizando Moodle.

\section{El entorno virtual gestionado por Biblioteca cuenta con 3 cursos claramente definidos:}

\subsection{Sesiones de Formación de Usuarios - "Conoce tu Biblioteca"}

Curso de inducción al catálogo, presentación de los productos y servicios que ofrece la biblioteca, consultas referidas al servicio, como plazos de préstamo de materiales, cómo realizar una reserva de materiales, cantidad de materiales que puede retirar, modalidades de préstamo, blog de Biblioteca, etc. No es un curso curricular por lo cual no genera créditos académicos y no es un curso de carácter obligatorio.

En este espacio virtual la Biblioteca ha adaptado los contenidos ofrecidos hasta 2010 en forma presencial al entorno virtual. Para ello ha generado Tutoriales, Videos y Material didáticos que explican y ejemplifican el uso del catálogo de Biblioteca y los recursos informativos. También se ha incorporado un Foro de consultas y un espacio con 
ejercicios breves para que el alumno pueda autoevaluarse, por ejemplo en el uso de los tipos de búsqueda que ofrece el catálogo.

Es importante destacar que al comienzo de todos los cursos se incluye un módulo de presentación de Moodle, explicando qué es, cómo funciona y se generan enlaces a manuales y videos de Moodle con el propósito que el alumno se familiarice con la herramienta recibiendo una breve inducción antes de iniciar cualquiera de los cursos propuestos. Este curso permanece abierto a todos los estudiantes del IUACJ durante todo el año.

\subsection{Ateneos académicos}

Este tipo de cursos dictados por Biblioteca son de carácter obligatorio y con un valor académico de 2 créditos. Los mismos están enfocados a alumnos de 1ำ, $2^{\circ}$ y $3^{\circ}$ año de la Licenciatura en Educación Física Recreación y Deporte. Los cursos son de carácter semipresencial, asignándole 4 horas a formación virtual. Se utilizan los Foros, para discutir temas tratados en el curso y generar debates académicos. También se utiliza el Enlace de archivos o web para la subida de materiales (manuales, tutoriales, artículos) relacionados con el curso en formato .pdf, o generando enlaces a sitios de interés, (p.e: instalación y manejo de gestores bibliográficos como Zotero, artículos en línea, etc).

Otros recursos que se utilizan son la aplicación de Cuestionarios de múltiple opción y las Tareas, indicándole en éste último ejercicios al estudiante en un período indicado por el docente para enviar la tarea, que luego es evaluada, corregida y calificada en la plataforma para que el alumno tenga acceso a las observaciones realizadas y al puntaje obtenido.

Se destaca que a pesar de asignar 4 horas a la modalidad virtual, se cargan todos los módulos del curso en la plataforma y se incluye información básica y ampliatoria en formato .pdf o direccionando al estudiante a enlaces o sitios de interés para que los alumnos pueden bajar y guardar o imprimir la información.

\subsection{Elaboración de Trabajos Académicos}

A continuación se describe la organización de la Licenciatura en Educación Física, Recreación y Deporte, con un valor académico de 2,5 créditos y una carga horaria de 25 horas; coordinada e impartida por personal de Biblioteca. Es una asignatura semipresencial la cual de un total de diez clases que componen el curso, cuatro clases se dictan a través de la plataforma.

Para el desarrollo del curso se utilizan los siguientes recursos que ofrece Moodle:

- Foro de Bienvenida (luego de la bienvenida y presentación del docente,cada alumnopara familiarizarse con la herramienta debe presentarse y subir una foto)

- Foro de Consultas en cada módulo

- Contenido teórico del curso (subida de archivos, artículos, videos, enlaces a sitios de interés, tutoriales, manuales, etc)

- Tareas

- Evaluación (se aplica una breve encuesta de valoración del curso y de la herramienta) 


\section{Conclusiones}

La Biblioteca del IUACJ valora positivamente la incorporación de herramientas como Moodle para el dictado de cursos dirigidos a estudiantes dado que se considera que facilita el acceso y la difusión del conocimiento académico en forma asincrónica.

La incorporación de esta herramienta permitió a la Biblioteca alcanzar más visibilidad y ejercer en forma más activa su rol alfabetizador, dado que la incorporación del entorno virtual, contribuyó positivamente a la tarea formadora para el desarrollo de conocimientos y habilidades de los usuarios.

Se potenció el trabajo colaborativo del estudiante, generando una participación más activa e instancias de trabajo cooperativo.

Se debe continuar trabajando en el perfeccionamiento y la formación continua del grupo responsable de llevar adelante los cursos, a fin de mantener la motivación del equipo y la satisfacción de los usuarios.

Cabe señalar que el desarrollo del entorno virtual de la Biblioteca del IUACJ fue posible gracias al apoyo y al trabajo multidisciplinario desarrollado con las Lic. Nancy Peré y Luciana Canutti de la Unidad Académica del Departamento de Apoyo Técnico Académico (DATA) de la Universidad de la República, quienes fueron responsables de llevar adelante la Consultoría en E-learning del Instituto Universitario de la Asociación Cristiana de Jóvenes y posteriormente los cursos de capacitación.

\section{Bibliografia}

CABERO ALMENARA, J. (2000) Las nuevas tecnologías al servicio del desarrollo de la Universidad: las teleuniversidades. En Rosales, C. Innovación en la Universidad, Santiago de Compostela, NINO, 187-216.

DÍAZ, M. L, ECHEVERRÍA, M.B, DELFINO, M.F. (2011) Orientación para la elaboración de las monografías: Espacio en el EVA.

GRUPO DE MEJORA DE FORMACIÓN DE USUARIOS. (2007). La formación de Usuarios en la Biblioteca de la Universidad de Málaga. En: XIV Jornadas Bibliotecarias de Andalucía. Málaga. PERÉ, N. (2010). Consultoría en e-learning. Informe de Relevamiento y Análisis de Plataformas.

PIAGET, J.P. (1952). The origins of intelligence in children. International University Press, New York. 


\section{BibliOS Information literacy through Moodle}

\section{Datos de la autora}

Gabriela Fernanda Cabrera Rossi

Directora de la Biblioteca Dr. Claudio Williman de la Facultad de Educación Física del Instituto Universitario de la Asociación Cristiana de Jóvenes, Montevideo - Uruguay. Responsable de la Biblioteca del Instituto de Ingeniería Eléctrica de la Facultad de Ingeniería, Universidad de la República (Uruguay).

gfcabrerarossi@gmail.com

$\begin{array}{ll}\text { Recibido-Received } & : 2013-07-19 \\ \text { Aceptado-Accepted } & : 2013-12-30\end{array}$

$(\mathrm{cc}) \mathrm{EY}$

This work is licensed under a Creative Commons Attribution 4.0

United States License.

\section{ULIS D-Sente}

This journal is published by the University Library System of the University of Pittsburgh as part of its D-Scribe Digital Publishing Program and is cosponsored by the University of Pittsburgh Press. 